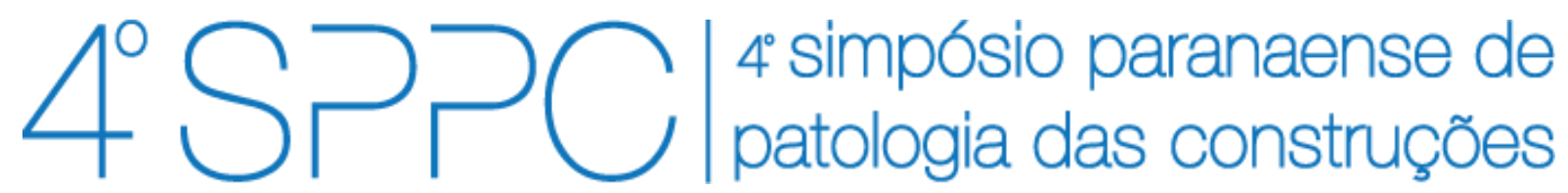

ISSN 2526-7248 artigo n. 4SPPC156, pp. 514-521, 2019

\title{
Efeito da substituição do cimento por cinza volante em concretos autoadensáveis de alto desempenho
}

\author{
Casagrande, Cézar A. ; Matos, Paulo R. ${ }^{2}$; Sakata, Rafael ${ }^{3}$; Jochem, Lidiane F. ${ }^{4}$. \\ ${ }^{1}$ Doutor, Universidade Federal de Pernambuco. Av. Campina Grande, s/n - Km 59 - Nova \\ Caruaru, Caruaru - PE, 55014-900, cezar.acasa@gmail.com; \\ ${ }^{2}$ Doutor, Universidade Federal de Santa Catarina. Departamento de Engenharia Civil, \\ paulorm.matos@gmail.com; \\ ${ }^{3}$ Doutorando, Universidade Federal de Santa Catarina. Departamento de Engenharia Civil, \\ rafaelsakata@gmail.com; \\ ${ }^{4}$ Doutora, Universidade Tecnológica Federal do Paraná. Departamento Acadêmico de \\ Construção Civil, lidijochem@gmail.com
}

Resumo: Avaliou-se concretos autoadensáveis de alto desempenho com substituição parcial de cimento por cinza volante em teores de 10, 20 e $30 \%$, em massa. Realizaram-se ensaios de resistência à compressão e de durabilidade de resistência a penetração de íons cloretos e de volume permeável aos 28 e 90 dias. Houve uma melhora nas propriedades mecânicas e de durabilidade das amostras em função da quantidade de cinza. Aumentou-se a resistência à compressão em todas as substituições em comparação com a referência. Houve diminuição dos índices de volume permeável em função da quantidade de cinza nas misturas. Além disso, com o aumento da quantidade de cinza volante, houve uma efetiva melhora na resistência aos íons cloretos, obtendo-se valores de menos da metade do valor de carga elétrica passante, com as amostras com adição de cinza em comparação com a amostra referência. A adição de cinza volante mostrou grande potencial para aprimorar as propriedades mecânicas e durabilidade de materiais cimentícios.

Palavras-chave: Concreto autoadensável, materiais cimentícios suplementares, durabilidade.

\begin{abstract}
High performance self-compacting concretes were evaluated with partial replacement of cement by fly ash in levels of 10,20 and $30 \%$ by mass. Tests were carried out by compression strength, durability by penetration resistance of chloride ions and porosity at 28 and 90 days. It was verified that there was an improvement in the mechanical properties and durability of the samples as a function of the amount of ash. Compressive strength was increased at all substitutions compared to the reference. There was a decrease in permeable volume indices as a function of the amount of fly ash in the mixtures. In addition, as the fly ash increased, there was an effective improvement in resistance to chloride ions, resulting in values of less than half the electric pasant charge value in the samples with addition, compared to the reference sample. The addition of fly ash showed great potential to improve the mechanical properties and durability of the cementitious materials.
\end{abstract}

Keywords: Self-compacting concretes, supplementary cementitious materials, durability. 


\section{Introdução}

Concretos de alto desempenho apresentam resistência à compressão na ordem dos 50 a $120 \mathrm{MPa}$ aos 28 dias de hidratação [1]. Além disso, apresentam altos índices de trabalhabilidade, normalmente sendo autoadensáveis. Por consequência, apresentam ótimas propriedades frentes à agentes agressivos, resultando em materiais com alta durabilidade, como alta capacidade de resistir à entrada de íons cloretos, ataque de sulfatos e carbonatação [2]. Apesar disso, o consumo de cimento para essa faixa de resistência costuma ser elevado em relação aos concretos convencionais. Dessa forma, uma visão mais ambientalmente correta para esse tipo de material vem sendo buscada nos mais recentes trabalhos. Considerando que para cada tonelada de cimento produzida, pelo menos outra tonelada de $\mathrm{CO}_{2}$ é gerada (por consequência da queima e subsequente moagem e transporte [3,4]), uma abordagem com menor consumo de cimento se faz necessária. Trabalhos vem mostrado a potencialidade de se utilizar materiais suplementares para a produção de concretos de alto desempenho e concretos auto adensáveis sem perda das propriedades mecânicas e com melhoras na durabilidade desses materiais $[5,6]$. Uma das adições mais amplamente empregadas são as cinzas volante e pesadas, metacaulim, fíler calcário, pós de porcelanato, areia de fundição entre outros pós/materiais com características pozolânicas [7-11]. Assim, neste trabalho foram desenvolvidos concretos auto adensáveis de alta resistência com a substituição parcial do cimento por uma cinza volante de alta reatividade, sendo verificados os seus efeitos frente as propriedades no estado endurecido, qualidade da matriz em relação à porosidade e frente à resistência à penetração aos íons cloretos.

\section{Materiais e métodos}

\subsection{Materiais}

Para a produção dos concretos, utilizou-se o cimento CP-V ARI, com massa específica de $3,14 \mathrm{~g} / \mathrm{cm}^{3}$, de acordo com a NBR 16697 [12]. Utilizou-se uma cinza volante de termoelétrica, de alta reatividade (moída), com massa específica de 2,36 $\mathrm{g} / \mathrm{cm}^{3}$, diâmetro médio de partículas $\left(d_{50}\right)$ de $14,40 \mu \mathrm{m}$ e $83 \%$ das partículas com potencial de reação pozolânica (isto é, $<30 \mu \mathrm{m}$ ), além do potencial de fixação de $518,5 \mathrm{~g}$ de $\mathrm{Ca}(\mathrm{OH})_{2} / \mathrm{g}$ de pozolana no ensaio de Chapelle modificado. Como agregado graúdo, utilizou-se brita com tamanho $<12,5 \mathrm{~mm}$, de massa específica $2,61 \mathrm{~g} / \mathrm{cm}^{3}$. Como agregado miúdo, utilizou-se uma composição de dois tipos de areia, uma areia natural de 2,65 $\mathrm{g} / \mathrm{cm}^{3}$ de massa específica e módulo de finura de 1,04 e uma areia artificial com massa espefícica de $2,80 \mathrm{~g} / \mathrm{cm}^{3}$ e módulo de finura de 3,03 . Utilizou-se também um aditivo superplastificante à base de policarboxilato (SP), com teor de sólidos de $39 \%$ e massa específica de $1,09 \mathrm{~g} / \mathrm{cm}^{3}$. Na Tabela 1 é apresentado 0 proporcionamento dos concretos estudados. Uma vez que o cimento foi substituído pela cinza volante em massa (a qual apresenta massa específica inferior à do cimento), e os consumos em massa de aglomerante e água por $\mathrm{m}^{3}$ de concreto foram mantidos, pode-se notar ligeiras diferenças nos consumos dos agregados.

O teor de aditivo utilizado foi em relação a massa de cimento. Todos os concretos foram dosados de maneira a obter um valor de espalhamento (Slump flow) de $700 \pm$ $25 \mathrm{~mm}$. 
CASAGRANDE, CÉZAR 1, CAC; MATOS, PAULO RICARDO 2, PRM; SAKATA, RAFAEL 3, RS; JOCHEM, LIDIANE 4, LFJ. EFEITO DA SUBSTITUIÇÃO DO CIMENTO POR CINZA EM CONCRETOS AUTOADENSÁVEIS DE ALTO DESEMPENHO. $4^{\circ}$ Simpósio

Paranaense de Patologia das Construções (40 SPPC), artigo 4SPPC156, pp. 514 - 521, 2019. DOI: 10.4322/2526-7248.073

Tabela 1: Consumo de materiais utilizados nos concretos $\mathrm{em} \mathrm{kg} / \mathrm{m}^{3} \mathrm{e}$ unitário

\begin{tabular}{cccccccc}
\hline Nomenclatura & Cimento & Cinza & Areia natural & $\begin{array}{c}\text { Areia } \\
\text { artificial }\end{array}$ & $\begin{array}{c}\text { Agregado } \\
\text { graúdo }\end{array}$ & Água & SP \\
\hline REF & $365(1)$ & 0 & $585(1,60)$ & $415(1,14)$ & $884(2,42)$ & $175(0,48)$ & $3,8(0,01)$ \\
$10 \%$ & $328(1)$ & $36(0,1)$ & $582(1,60)$ & $413(1,13)$ & $879(2,41)$ & $175(0,48)$ & $2,2(0,01)$ \\
$20 \%$ & $292(1)$ & $73(0,1)$ & $579(1,59)$ & $411(1,13)$ & $875(2,40)$ & $175(0,48)$ & $2,2(0,01)$ \\
$30 \%$ & $255(1)$ & $109(0,1)$ & $576(1,58)$ & $409(1,12)$ & $871(2,39)$ & $175(0,48)$ & $1,9(0,01)$ \\
\hline
\end{tabular}

\subsection{Métodos}

Todos os ensaios foram preparados e realizados para 28 e 90 dias de hidratação do cimento no Laboratório de Materiais de Construção Civil da Universidade Federal de Santa Catarina. Para a determinação do volume de vazios permeáveis, utilizou-se a metodologia da ASTM C642 [13], com corpos de prova de $100 \mathrm{~mm}$ de diâmetro e 50 $\mathrm{mm}$ de altura.

Para o ensaio de resistência à compressão, utilizou-se corpos de prova de $100 \mathrm{~mm}$ de diâmetro por $200 \mathrm{~mm}$ de altura, curados em sala climatizada com $100 \%$ de umidade relativa até o dia do ensaio. Os ensaios foram realizados de acordo com a ASTM C39 [14], para cada ensaio, 2 corpos de prova foram ensaiados.

Para a determinação da resistência à penetração aos íons cloretos, foi realizado um ensaio acelerado de acordo com a ASTM C1202 [15]. Pelo menos 3 corpos de prova foram ensaiados para esse parâmetro, adotando os valores médios.

\section{Resultados e discussão}

$\mathrm{Na}$ Figura 1, são apresentados os resultados de variação do índice de vazios permeáveis na matriz em função da quantidade de cinza volante incorporada ao concreto, aos 28 e 90 dias de hidratação. É possível verificar que, para ambos os tempos de hidratação, o volume de vazios é reduzido a medida que a quantidade de cinza incorporada em substituição ao cimento Portland é aumentada. Vale notar que, em todas as amostras avaliadas, os valores de 28 e 90 dias não obtiveram diferenças significativas. Isto é, depois da cura do material, pouca evolução meso/macroestrutural pode ser verificada. Isso significa que as maiores mudanças na matriz cimentícia acontecem antes dos 28 dias de hidratação. Este comportamento era esperado, pois o cimento empregado (de alta resistência inicial) apresenta na sua composição grande quantidade de $\mathrm{C}_{3} \mathrm{~S}$, que apresenta taxas de hidratação na ordem dos $70-90 \%$ até o 28 dia de hidratação, enquanto que o $\mathrm{C}_{2} \mathrm{~S}$ (pouco presente neste tipo de cimento) apresenta hidratação lenta nesse mesmo período [16]. A maior diferença de vazios entre amostras foi obtida entre 0 e $10 \%$ de substituição por cinza volante, é possível verificar uma redução de $11,5 \%$ na amostra referência para cerca de $8,9 \%$ para amostra com $10 \%$ de cinza, enquanto que para as amostras com 20 e $30 \%$ resultou em valores de 8 e $7 \%$, respectivamente. Com isso é possível perceber que há grande benefício da incorporação de cinzas na maior densificação da matriz, porém esse efeito não é linear e tende a ter menor eficiência quanto maior a quantidade de cinza adicionada. Isso é previsto, uma vez que nem toda a cinza atua como pozolana na reação de hidratação, resultando apenas em efeito fíler, que apresenta menos capacidade de preenchimento da estrutura, em relação a precipitação de C-S-H. 


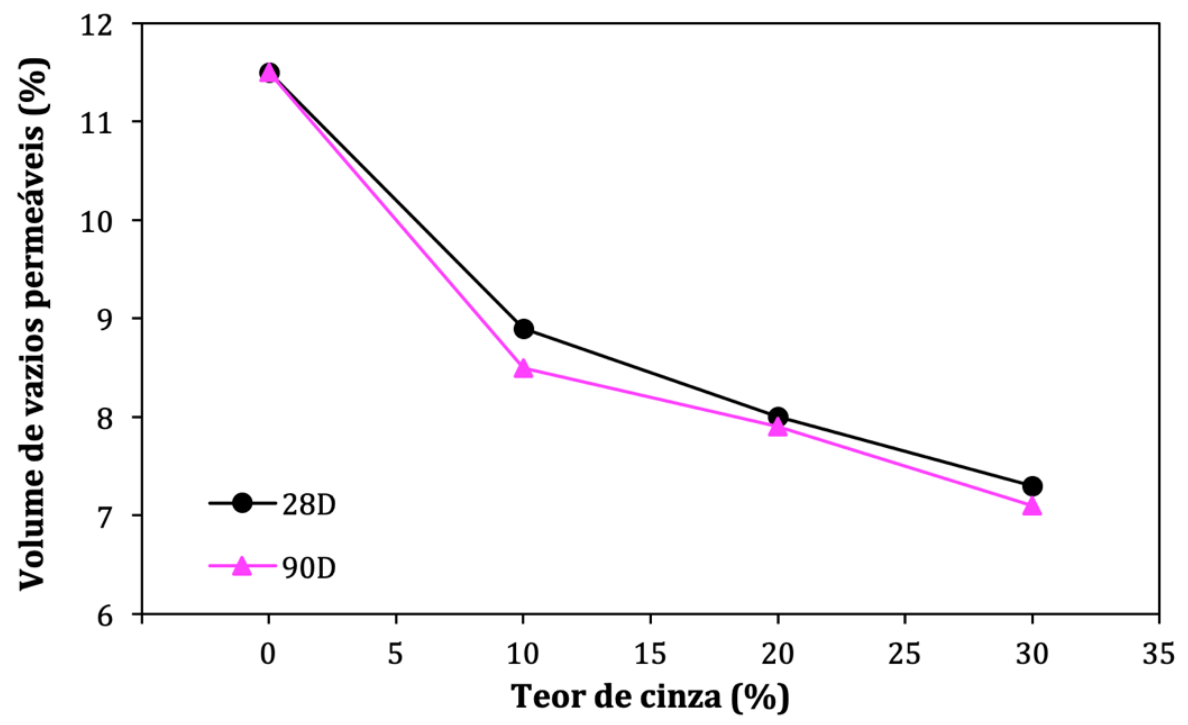

Figura 1: Influência do teor de cinza volante nos poros permeáveis da matriz cimentícia.

Na Figura 2 são apresentados os resultados de resistência à compressão dos concretos. Em geral, quando incorporada cinza em substituição ao cimento, houve um aumento nas propriedades mecânicas do compósito. Isso está relacionado também com a diminuição da porosidade, indicada anteriormente na Figura 1. Aqui o que se nota é que, aos 28 dias de hidratação, houve aumentos na resistência à compressão com a adição da cinza, em comparação à referência. Entretanto, o teor de $10 \%$, apresentou a maior resistência à compressão entre os concretos. Isso pode ser justificado pela quantidade de hidróxido de cálcio na solução dos poros da pasta cimentícia: aos 28 dias, a presença $10 \%$ de cinza já é suficiente para consumir todo o hidróxido de cálcio que possui potencial de reação pozolânica. Assim, quantidades superiores de cinza não deverão reagir (isto é, apresentar atividade pozolânica), resultando apenas em efeito fíler, (nesse caso, os $10 \%$ já estariam cumprindo também esse papel). Outra justificativa seria o fato de estar reduzindo-se a quantidade de cimento, que é o principal agente aglomerante do compósito. Dessa forma, espera-se que não haja aumento das propriedades mecânicas além de certo ponto da substituição de cimento por outro material, como verificado. Já, aos 90 dias, o que se verifica é um aumento progressivo da resistência mecânica, quanto maior a quantidade de cinza na matriz cimentícia. Esse fenômeno pode ser justificado pela evolução da hidratação do cimento, que apesar de menos intensa que nos momentos iniciais, é contínua e resulta em mais precipitação de hidróxido de cálcio com potencial de reação com a cinza. Isso densifica a matriz cimentícia através da precipitação do C-S-H resultante da reação pozolânica. Isso, resulta em um desequilíbrio do pH da solução básica da pasta, que é reduzida devido ao consumo do hidróxido de cálcio pela reação pozolânica. Esse desiquilíbrio induz uma dissolução nos produtos anidros, que ainda resultam na matriz cimentícia, produzindo maior quantidade de produtos hidratados e potencializando as propriedades mecânicas do compósito, como verificado. No caso estudado, é possível verificar que, mesmo com o aumento progressivo da resistência em função da adição de cinzas, há um ponto de saturação em torno dos $30 \%$ de substituição, de modo que um aumento deste teor de 20 para $30 \%$ resultou no aumento da resistência à compressão de 70 para $71 \mathrm{MPa}$ em valores médios. Isso caracteriza que valores maiores que $20 \%$ em substituição podem não ser mais eficientes quando busca-se o aumento das propriedades mecânicas. Por 
CASAGRANDE, CÉZAR 1, CAC; MATOS, PAULO RICARDO 2, PRM; SAKATA, RAFAEL 3, RS; JOCHEM, LIDIANE 4, LFJ. EFEITO

DA SUBSTITUIÇÃO DO CIMENTO POR CINZA EM CONCRETOS AUTOADENSÁVEIS DE ALTO DESEMPENHO. $4^{\circ}$ Simpósio

Paranaense de Patologia das Construções (40 SPPC), artigo 4SPPC156, pp. 514 - 521, 2019. DOI: 10.4322/2526-7248.073

outro lado, quando se analisa a questão ambiental dos materiais cimentícios, quanto menor o consumo de cimento/ $\mathrm{m}^{3}$ de concreto, menor é a geração de $\mathrm{CO}_{2}$ para a produção desse volume de concreto, sendo mais ecologicamente eficiente.

Do ponto de vista ecológico, $30 \%$ de substituição apresentou o maior potencial e eficiência dentre as substituições estudadas, uma vez que não apresentou perda significativas das propriedades mecânicas e apresentou um menor consumo de cimento dentre todas as amostras testadas.

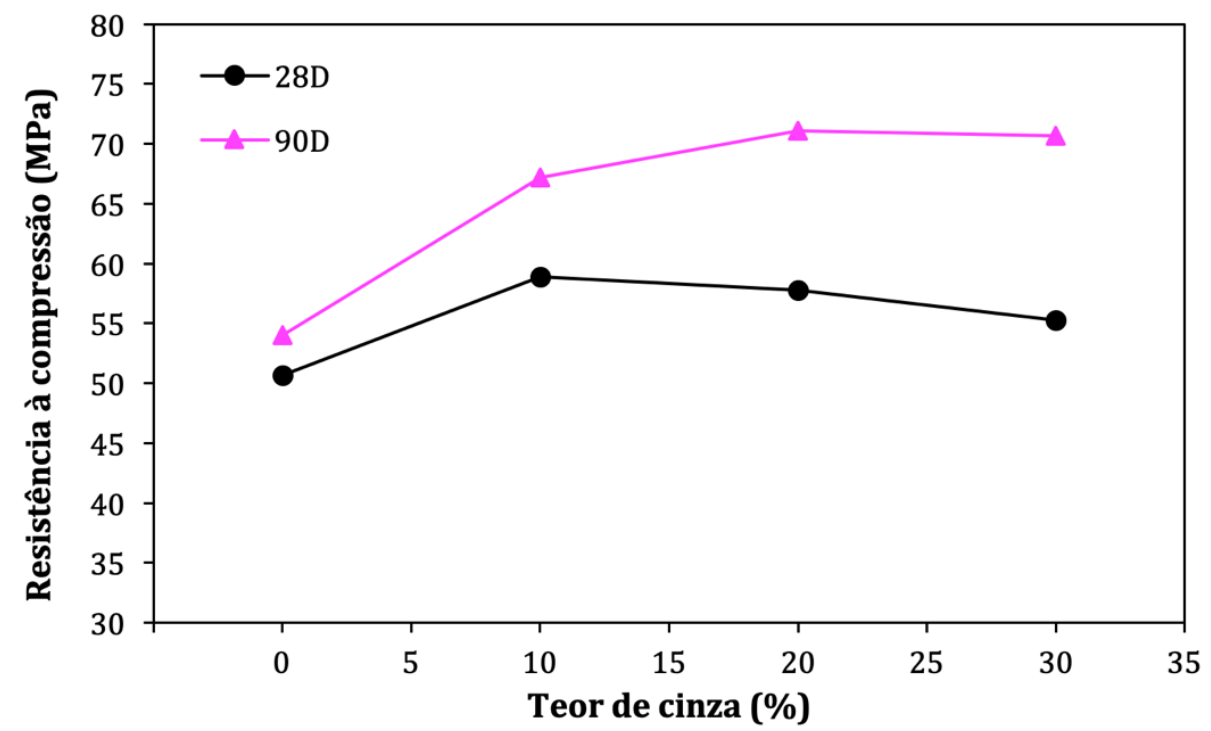

Figura 2: Efeito do teor de cinza volante na resistência mecânica à compressão dos concretos

Na Figura 3 são apresentados os valores de carga elétrica passante no ensaio de resistência aos íons cloretos. É possível verificar que houve uma grande diferença de resultados aos 28 e 90 dias. Apenas para a amostra referência não houve diferença nos valores em função do tempo de hidratação do cimento, o que está relacionada com a não variação dos valores obtidos de porosidade. Já, as demais amostras apresentaram uma grande redução de carga passante em função do aumento do teor de cinza e da evolução da hidratação. Isso está relacionado à dois fatores: Um deles é o fator físico, que é o aumento da compacidade e preenchimento dos poros pela adição da cinza (que resulta em maior grau de hidratação pela precipitação de produtos hidratados resultantes das reações pozolânicas); o outro é a maior hidratação do cimento, devido a alteração do pH e consumo de hidróxido de cálcio, que resultam em uma barreira física para os íons dissolvidos.

Esses íons cloretos dissolvidos nos poros precisam difundir através da matriz cimentícia para a passagem de carga elétrica, que é dificultado pela menor conectividade dos poros, que foram preenchidos por cinza ou foram preenchidos por matriz cimentícia. Outro fator é a mudança de íons dissolvidos na solução dos poros da matriz, que altera as cinéticas de dissolução/precipitação dos compostos da matriz cimentícia e altera a cinética de difusão dos íons cloretos, resultando na menor capacidade de carga elétrica passante observada. 
CASAGRANDE, CÉZAR 1, CAC; MATOS, PAULO RICARDO 2, PRM; SAKATA, RAFAEL 3, RS; JOCHEM, LIDIANE 4, LFJ. EFEITO DA SUBSTITUIÇÃO DO CIMENTO POR CINZA EM CONCRETOS AUTOADENSÁVEIS DE ALTO DESEMPENHO. $4^{\circ}$ Simpósio Paranaense de Patologia das Construções (40 SPPC), artigo 4SPPC156, pp. 514 - 521, 2019. DOI: 10.4322/2526-7248.073

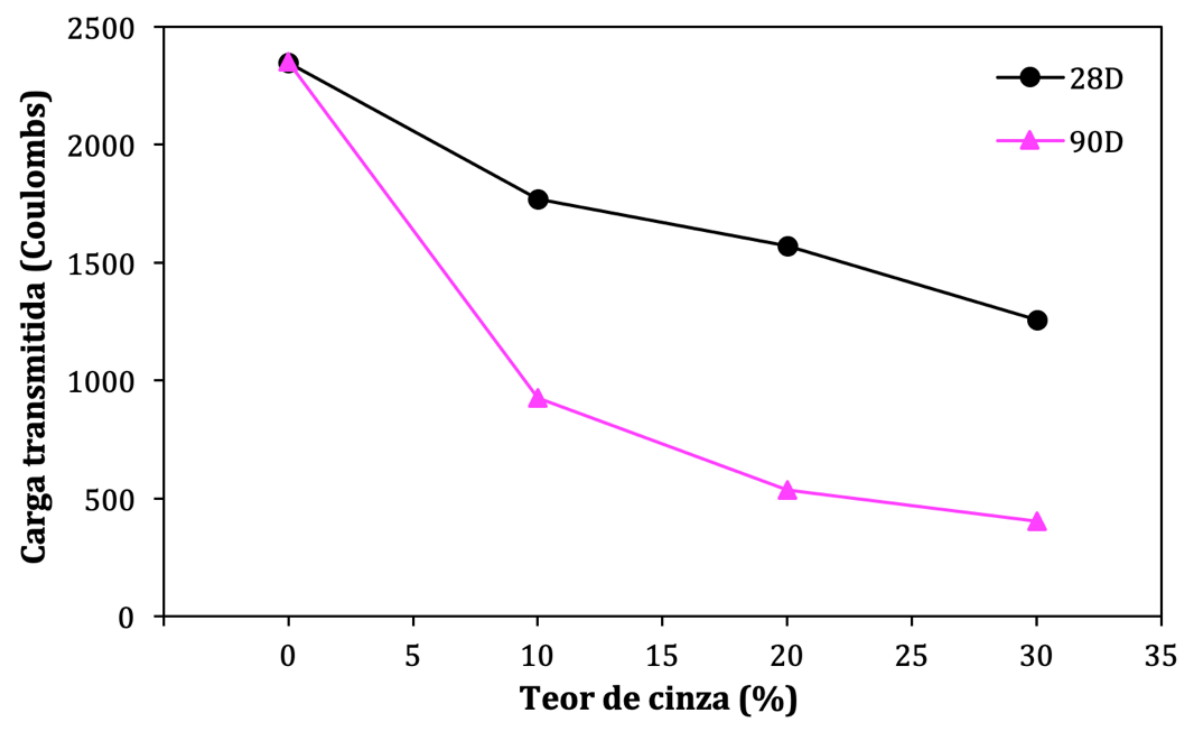

Figura 3: Variação da resistência aos íons cloretos em função da quantidade de cinza volante incorporada na matriz

Na Figura 4 é apresentada uma correlação da carga elétrica passante no ensaio de resistência aos íons cloretos em função do volume de vazios permeáveis. Essa correlação foi realizada pois o volume de vazios está diretamente relacionado com a durabilidade dos materiais cimentício. Fica evidente a relação dessas duas propriedades com os valores os coeficientes correlação $\mathrm{R}^{2}$ obtidos para ambas as idades avaliadas. Tanto com 28 quanto para 90 dias, é possível verificar que há uma diminuição linear, com $\mathrm{R}^{2}$ na ordem de 0,98 , que evidencia uma ótima correlação entre as propriedades analisadas. Como abordado, o aumento do impedimento físico oriundo da maior densificação da matriz cimentícia promovida pela adição da cinza resulta em uma menor porosidade e, consequentemente, menor capacidade de transmissão de massa (nesse caso, íons cloretos), que pode ser relacionado diretamente com uma alta durabilidade.

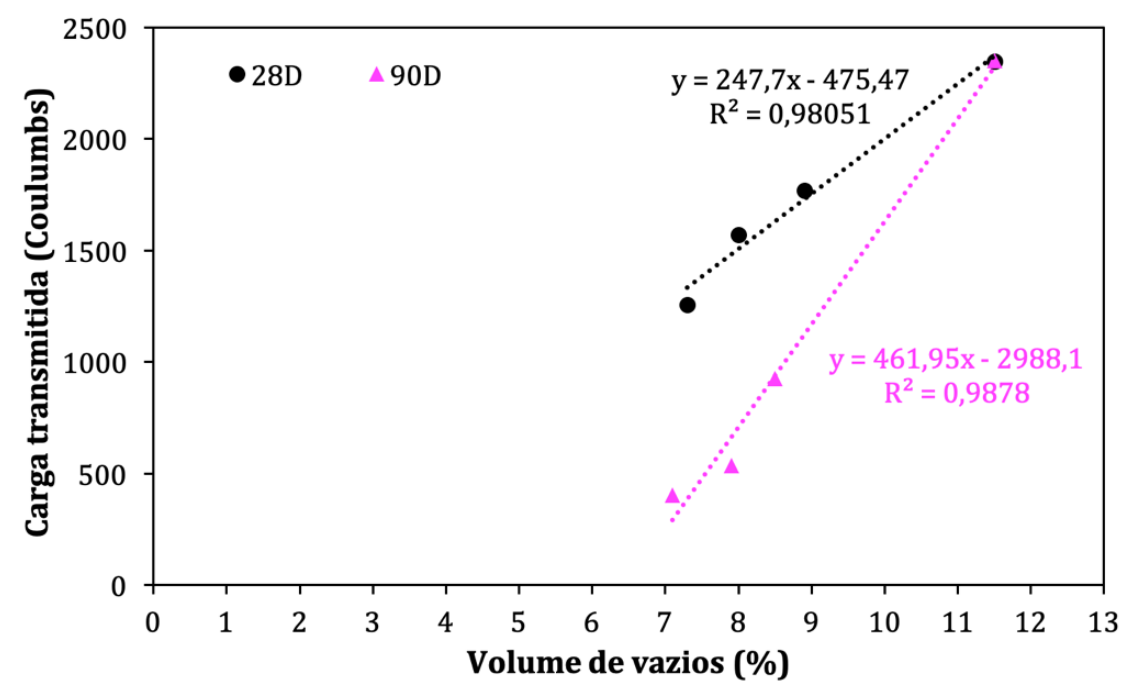

Figura 4: Correlação da carga elétrica passante em função do volume de vazios da matriz cimentícia. 
CASAGRANDE, CÉZAR 1, CAC; MATOS, PAULO RICARDO 2, PRM; SAKATA, RAFAEL 3, RS; JOCHEM, LIDIANE 4, LFJ. EFEITO

DA SUBSTITUIÇÃO DO CIMENTO POR CINZA EM CONCRETOS AUTOADENSÁVEIS DE ALTO DESEMPENHO. 40 Simpósio

Paranaense de Patologia das Construções (40 SPPC), artigo 4SPPC156, pp. 514 - 521, 2019. DOI: 10.4322/2526-7248.073

\section{Conclusões}

Neste trabalho foram avaliados, experimentalmente, concretos autoadensáveis de alto desempenho com substituição parcial de cimento por uma cinza volante de alta reatividade. Através dos resultados obtidos é possível concluir que:

- Quanto maior a quantidade de cinza volante de alta reatividade na mistura cimentícia, maiores foram os resultados de resistência à compressão promovida por meio de efeito fíler e através da atividade 5200zolânicas, resultando em maior compacidade da matriz;

- Quanto maior a quantidade de cinza volante, menores foram os valores de volume permeável (porosidade) obtido nas amostras, tanto para 28 quanto para 90 dias. Da mesma forma, os efeitos causadores dessa diminuição da porosidade são os preenchimentos dos poros com cinza e maior precipitação do C-S-H na microestrutura do material;

- Os concretos com maior quantidade de cinza na mistura apresentaram menores valores de carga elétrica passante no ensaio de penetração acelerada de íons cloreto, o que indica maior durabilidade frente à agentes agressivos, em comparação com a amostra referência.

\section{Agradecimentos}

Os autores gostariam de agradecer às agências de fomento CAPES e CNPQ pelo financiamento de bolsas de estudo e pelo financiamento dos laboratórios de pesquisa da Universidade Federal de Santa Catarina.

\section{Referências}

[1] P.K. Mehta, P.J.M. Monteiro, Concreto: Microestrutura, Propriedades e Materiais, Segunda, IBRACON, São Paulo, Brasil, 2014.

[2] S.S. Vivek, G. Dhinakaran, Durability characteristics of binary blend high strength SCC, Constr. Build. Mater. $146 \quad$ (2017) 1-8. doi:10.1016/J.CONBUILDMAT.2017.04.063.

[3] K.P. Mehta, Reducing the Environmental Impact of Concrete, Concr. Int. 23 (n.d.).

[4] M.B. Ali, R. Saidur, M.S. Hossain, A review on emission analysis in cement industries, Renew. Sustain. Energy Rev. 15 (2011) 2252-2261. doi:10.1016/J.RSER.2011.02.014.

[5] K.L. Scrivener, R.J. Kirkpatrick, Innovation in use and research on cementitious material, Cem. Concr. Res. 38 (2008) 128-136. doi:10.1016/j.cemconres.2007.09.025.

[6] W. Huang, H. Kazemi-Kamyab, W. Sun, K. Scrivener, Effect of cement substitution by limestone on the hydration and microstructural development of ultra-high performance concrete (UHPC), Cem. Concr. Compos. 77 (2017) 86101. doi:10.1016/j.cemconcomp.2016.12.009. 
CASAGRANDE, CÉZAR 1, CAC; MATOS, PAULO RICARDO 2, PRM; SAKATA, RAFAEL 3, RS; JOCHEM, LIDIANE 4, LFJ. EFEITO DA SUBSTITUIÇÃO DO CIMENTO POR CINZA EM CONCRETOS AUTOADENSÁVEIS DE ALTO DESEMPENHO. $4^{\circ}$ Simpósio

Paranaense de Patologia das Construções (40 SPPC), artigo 4SPPC156, pp. 514 - 521, 2019. DOI: 10.4322/2526-7248.073

[7] P.R. de Matos, A.L. de Oliveira, F. Pelisser, L.R. Prudêncio, Rheological behavior of Portland cement pastes and self-compacting concretes containing porcelain polishing residue, Constr. Build. Mater. 175 (2018) 508-518. doi:10.1016/j.conbuildmat.2018.04.212.

[8] P.R. De Matos, A.L. De Oliveira, F. Pelisser, P. Jean, P. Gleize, Use of porcelain polishing residue as a supplementary cimentitious material in self-compacting concrete, Constr. Build. Mater. In press (2018) 623-630. doi:10.1016/j.conbuildmat.2018.10.228.

[9] P.R. de Matos, M.F. Marcon, R.A. Schankoski, L.R. Prudêncio Jr., Novel applications of waste foundry sand in conventional and dry-mix concretes, J. Environ. Manage. 244 (2019) 294-303. doi:10.1016/j.jenvman.2019.04.048.

[10] R. Yu, P. Spiesz, H.J.H. Brouwers, Development of an eco-friendly Ultra-High Performance Concrete (UHPC) with efficient cement and mineral admixtures uses, Cem. Concr. Compos. $55 \quad$ (2015) 383-394. doi:10.1016/j.cemconcomp.2014.09.024.

[11] M. Gesoglu, E. Güneyisi, D.S. Asaad, G.F. Muhyaddin, Properties of low binder ultra-high performance cementitious composites: Comparison of nanosilica and microsilica, Constr. Build. Mater. $102 \quad$ (2016) 706-713. doi:10.1016/j.conbuildmat.2015.11.020.

[12] Associação Brasileira de Normas Técnicas, NBR 16697 - Cimento Portland Requisitos, Rio de Janeiro, 2018. http://www.abntcatalogo.com.br/norma.aspx?ID=400221.

[13] American Society of Testing Materials, ASTM C 642-13 - Standard Test Method for Density , Absorption, and Voids in Hardened Concrete, West Conshohocken, 2013. doi:10.1520/C0642-13.

[14] American Society of Testing Materials, ASTM C39-18 - Standard Test Method for Compressive Strength of Cylindrical Concrete Specimens, West Conshohocken, 2018. doi:10.1520/C0039_C0039M-18.

[15] American Society of Testing Materials, ASTM C1202 - Standard Test Method for Electrical Indication of Concrete's Ability to Resist Chloride lon Penetration, West Conshohocken, 2019. doi:10.1520/C1202-19.

[16] J. Bensted, R.G. Blezard, B. Brown, A. Capmas, R.M. Edmeades, P. Fidjestol, F.P. Glasser, P.J. Jackson, D.C. Lawrence, R. Lewis, D.E. Macphee, F. Massazza, M. Moranville-Regourd, I. Odler, K.L. Scrivener, I. Sims, Lea's Chemistry of Cement and Concrete, Fourth, Elsevier Science \& Technology Books, Oxford, UK, 2004. 